\title{
$\checkmark$ Research Square \\ Striving for Sufficient Milk to Have a Healthy Late Preterm Baby: a Grounded Theory Study
}

\section{Rungtawan Choijorhor}

Chiang Mai University Faculty of Nursing

\section{Kannika Kantaruksa}

Chiang Mai University Faculty of Nursing

Jutamas Chotibang ( $\nabla$ chotibang.coact@gmail.com )

Chiang Mai University Faculty of Nursing https://orcid.org/0000-0002-2524-949X

\section{Nonglak Chaloumsuk}

Chiang Mai University Faculty of Nursing

\section{Research Article}

Keywords: Exclusive breastfeeding, Late premature infant, Lactation management, Grounded theory,

Thailand

Posted Date: March 1st, 2022

DOI: https://doi.org/10.21203/rs.3.rs-342392/v2

License: (c) (1) This work is licensed under a Creative Commons Attribution 4.0 International License. Read Full License 
1 Title "Striving for sufficient milk to have a healthy late preterm baby: A grounded theory study"

2 Rungtawan Choijohor ${ }^{1,2}$, Kannika Kantaruksa ${ }^{1}$, Juthamas Chotibang ${ }^{1,3^{*}}$ and Nonglak

3 Chaloumsuk ${ }^{1}$

$4 \quad{ }^{1}$ Faculty of Nursing, Chiang Mai University, Chiang Mai, 50200, Thailand

$5 \quad 2$ Boromarajonani College of Nursing, 10400, Bangkok, Thailand

$6{ }^{3}$ Center of Excellence in Nursing, Chiang Mai University, Chiang Mai, 50200, Thailand

7

8 Corresponding author:

9 Jutamas Chotibang, RN, $\mathrm{PhD}$

10 Faculty of Nursing, Chiang Mai University, 110/406 Intrawaroros Road, Suthep District,

11 Mueang, Chiang Mai, 50200, Thailand.

12 Tel: 66-53-93-5024, Fax: 66-53-21-7145, E-mail: jutamas.c@cmu.ac.th.

13

14

15

16

17

18

19

20

21

22

23

24

25

26

27

28 
Background: Late preterm infants are at risk for more health problems than full-term infants.

They require good nutrition for growth, and breast milk is the gold standard because it contains valuable nutrients. Currently, the population of late preterm infants is increasing as exclusive breastfeeding rates decrease. Hence, this study explored exclusive breastfeeding experiences among Thai first-time mothers of late preterm infants.

Methods: Participants were recruited from the family planning unit of a university hospital in Northern Thailand. A grounded theory study design was adopted, and an interview guideline was used to interview seventeen first-time mothers who exclusively breastfed their late preterm infants for the first six weeks of life.

Results: "Striving for sufficient milk to have a healthy baby" emerged as the core category. It was defined as a process in which mothers of late preterm infants had to exert great effort to

41 produce sufficient milk for their infants. The following three phases supported the core category:

42 preparing for breastfeeding, overcoming the problem of insufficient milk supply, and managing 43 to continue breastfeeding.

44 Conclusion: Perceived insufficient milk supply in first-time mothers is a threat to achieving 45 exclusive breastfeeding of their late preterm infants. However, the desire to have a healthy baby 46 results in mothers' effort to achieve a sufficient milk supply. Nurse midwives could apply the 47 management process of exclusive breastfeeding as a guideline when providing support for these 48 mothers throughout the sequence of antenatal, intrapartum, and postpartum care. Trial registration: Permission to conduct the study was obtained from the Institutional Review 50 Board Committee, Faculty of Nursing, and the Faculty of Medicine, Chiang Mai University, No. 51 2561-EXP065 and No. 2561-05865.

52 Keywords: Exclusive breastfeeding, Late premature infant, Lactation management, Grounded 53 theory, Thailand 
Late preterm infants (LPIs), defined by birth at $34^{0 / 7}$ through $36^{6 / 7}$ weeks' gestation, are less physiologically and metabolically mature than term infants. Thus, they are at risk for more

57 health problems and have higher death rates than full-term infants [7]. Breast milk is the ideal food for optimal nutrition and growth in preterm infants [29]. Mothers of preterm infants initially produce breast milk with higher amounts of protein, fat, free amino acids, and sodium than those

60

61 of full-term infants [20]. Regarding development, breast milk contributes positively to physical, neurological, and psychological development in preterm infants [18].

Although breastfeeding is beneficial for all infants, the exclusive breastfeeding rate is lower than the global requirement [31]. The WHO set a goal for an exclusive breastfeeding rate in infants-both full-term and preterm - of $50 \%$ by 2025 [30]. In Thailand, a target rate of $50 \%$ for exclusive breastfeeding was also set in the Twelfth National Health Development Plan [5]. However, Thailand has not reached that target. The exclusive breastfeeding rate for all infants in Thailand is $23.1 \%$ [24]. Currently, the rate of preterm birth is still increasing. Late preterm births account for more than $70 \%$ of all preterm births [23]. As a consequence, late preterm infants are less likely to be breastfed than term infants [14]. Due to the high mortality rates of preterm infants and the low rates of exclusive breastfeeding, the need to study how mothers can continue to breastfeed to ensure the survival of their late preterm children is an urgent issue.

Though there are studies on the experiences of mothers of LPIs, these studies were conducted with mothers of various parities who had LPIs with complications staying in the NICU. Additionally, the exclusive breastfeeding experiences and the management of successful exclusive breastfeeding in first-time mothers of LPIs have not been sufficiently studied. According to a literature review, few academic studies have focused on the exclusive breastfeeding experiences of first-time mothers of LPIs. Hence, the exclusive breastfeeding experiences of Thai first-time mothers of LPIs should be explored. 
To explore the exclusive breastfeeding experiences of Thai first-time mothers of LPIs,

the researchers conducted a grounded theory study [12]. The investigation used symbolic interactionism (SI), which focuses on the processes of interaction between people, exploring behavior and social roles [15]. Therefore, this grounded theory approach can be used to explore the meaning of breastfeeding experiences among mothers of LPIs and the breastfeeding management processes that facilitate success in exclusive breastfeeding. It is particularly suited to areas of this research that had not previously been explored. Moreover, this approach allows the emergence of theory from research material that could be explained by human behavior and social contexts [11].

The researchers studied mothers at 6 weeks postpartum because this period is critical for the establishment of exclusive breastfeeding [27], and a mother's ability to breastfeed at 6 weeks is a significant predictor of exclusive breastfeeding for up to 6 months [26]. In addition, a followup at 6 weeks postpartum can reveal the management of breastfeeding among Thai mothers of LPIs while in the hospital, until discharge and at home [6]. The aim of this study is to describe exclusive breastfeeding experiences among first-time mothers of LPIs and explore how they manage to exclusively breastfeed their LPIs.

\section{Methods}

\section{Research design}

A grounded theory approach is used in this study to develop or create a theory from the research process via the inductive method, that is, conclusion drawn through collection and systematic analysis of data derived from the studied phenomenon. The process for this research involves a method of simultaneously collecting and analyzing qualitative research data by providing codification to categorize the data, select core category, and define relationships with other categories to develop a rational relationship model or theoretical diagram. A grounded 
theory approach enables researchers to describe how individuals interpret objects and other people in their lives and how this process of interpretation leads to certain behaviors in certain contexts [12].

\section{Participants and setting}

A purposive sampling strategy and theoretical sampling were used in this study. Seventeen first-time mothers of LPIs who had delivered at gestational ages of $34-36^{+6}$ weeks were eligible to participate if they met the following inclusion criteria, in order to ensure homogeneity of study participants: a) 20 years of age or older, b) exclusively breastfeeding, c) normal delivery, d) no underlying diseases, e) Thai-speaking, and f) having a healthy infant without congenital abnormalities and exclusively breastfeeding for the first six weeks of life. The researchers selected mothers who were exclusively breastfeeding in order to analyze their experience and how they managed to exclusively breastfeed successfully. Enrollment occurred when the participants came for a six-week postpartum checkup at the family planning clinic of a university hospital in Northern Thailand. After a mother agreed to participate in the research, the researcher made appointments and set up dates, times, and private places for the interviews that were convenient for the patient. The in-depth interviews were conducted in the family planning clinic room.

\section{Data collection}

The first author (RC), who was not an employee of the unit or hospital, collected all the data between November 2018 and September 2019. RC had no prior contact or relationships with the research participants prior to initiation of the study. The first author conducted an individual, face-to-face interview with each participant in the family planning clinic room at the study site using an interview guide to obtain intensive descriptions of experience related to the 

and grounded theory study. It included opened-ended questionnaires. The interviews inquired about the experiences and management of exclusive breastfeeding among mothers of late

132 preterm infants during pregnancy, giving birth and postpartum period. The researcher submitted 133 this guide to two breastfeeding experts and one expert in grounded theory methodology to 134 examine the properties of the content and the language used in the instrument. After developing 135 an interview guide, the researcher tested the guide before collecting data. All interviews were 136 conducted by the first author, lasted an average of one hour, and were audio recorded and 137 transcribed verbatim. Table 1 provides examples of the questions asked during the interviews. 138 After several pro forma, general questions establishing identity, the researcher opened with a 139 broad research question: "Can you tell me about your breastfeeding experience?" During the 140 interviews, the researcher probed more deeply into specific issues the participants had introduced 141 in order to gain deeper and clearer information, using expressions such as "Please tell more 142 about..." and "How?" The researchers used an audio recorder to collect the data. The interviews 143 lasted from 30 to 60 minutes. The field notes were made after the interview. The researcher then 144 wrote theoretical memos and transcribed the entire recording. To ensure accuracy after the 145 interviews, each participant received a written summary of her first interview to review. There 146 were no changes requested by the participants. All participants were interviewed twice in the 147 interest of thoroughness and academic accuracy. The data was continually collected until it is 148 saturated [13].

150 Ethical considerations

Permission to conduct the study was obtained from the Institutional Review Board 152 Committee, Faculty of Nursing, and the Faculty of Medicine, Chiang Mai University, No. 2561153 EXP065 and No. 2561-05865. The potential participants were informed about the purpose and 
154 process of the research and ensured, with oral and written guarantees, anonymity and 155 confidentiality. It was clarified that they could refuse to participate or withdraw from the study at 156 any time. All participants signed informed consent forms, which included permission to record 157 the interviews. All interview transcripts were kept confidential and anonymous, and only the 158 advisory committee could check them for review purposes.

159

160

161

162

164

165

166

167

168

169

170

171

172

173

174

175

176

177

178

\section{Data analysis}

Data collection and analysis were performed simultaneously using the three steps of data analysis described by Glaser (1998), which involves a constant comparative method during the coding procedure, memos with diagrams, and theoretical sensitivity [13]. Codes were identified in the initial phase by using open, line-by-line, and incident-by-incident coding, followed by selective, focused, and theoretical coding. The topic codes were grouped to form categories. In the memos-with-diagrams step, each category was linked and compared with the other categories to verify the findings and the categories to ensure that these categories fit the data by using the constant comparative method. Finally, the researcher used theoretical sensitivity to assist in the formulation of a theory that was specific to the phenomena under study [12]. Following analysis, member checking was used to ascertain whether the participants recognized the research findings to be true to their experiences. The researcher revisited the participants to ensure that the interpretation of their responses suitably reflected their thoughts about their experience. In the peer debriefing process, the first three interviews were coded and discussed with the advisory committee. The data analysis was supervised by a committee consisting of two experts in grounded theory, three experts in breastfeeding and one expert in preterm infant care throughout the research inquiry process. 


\section{Trustworthiness of the study}

Member checking with participants and peer debriefing ensured credibility. In addition, the researchers used thick description, purposive sampling and theoretical sampling to judge the transferability potential. The research team regularly checked in with the participants to ensure that they understood the research process and to address any concerns they might have. The data analysis was regulated by the committee throughout the research inquiry process. The researchers' conceptualizations were discussed with two grounded theory experts, two experts in breastfeeding, and one expert in preterm infants. Through these means, external credibility was strengthened. Agreement pertained to the emerging categories and the core category. A summary of the emerging themes was given to participants to determine whether the codes and categories matched their perspectives.

\section{Results}

\section{Participant characteristics}

The participants were 17 Thai first-time mothers of LPIs. Of the mothers, $88.24 \%$ lived in extended families; $52.94 \%$ gave birth at $35-35^{+6}$ weeks of gestation; $70.59 \%$ of the infants were transferred to the neonatal ward (NS2 unit) and $29.41 \%$ were transferred to the neonatal intensive care unit (NICU); and $64.71 \%$ of the infants were given breast milk at 6 weeks postpartum by breastfeeding, while $32.29 \%$ were given breast milk by bottle feeding (Table 2).

\section{Core Category}

Process of "Striving for sufficient milk to have a healthy baby"

The data analysis revealed that "Striving for sufficient milk to have a healthy baby" was a process in which the participants had to exert great effort to secure sufficient milk for their infants. This process is a basic social process in the life journey that begins when participants 
204 plan to give breast milk to their babies during pregnancy and lasts until they achieve

205 breastfeeding, which continues at 6 weeks postpartum. The process consists of the following

206 three phases: (1) preparing for breastfeeding; (2) overcoming the problem of insufficient milk

207 supply; and (3) managing to continue breastfeeding (Fig. 1).

208 Phase 1: Preparing for breastfeeding

209

210

211

212

213

214

215

216

217

218

219

220

221

222

223

224

225

226

227

The mothers began preparing for breastfeeding from while they were pregnant until they delivered their preterm babies. As all participants received antenatal care services from the hospital, they received information on the benefits of breastfeeding from professionals, particularly nurses at the antenatal clinics, through group learning sessions. Therefore, all participants made the decision to breastfeed prenatally, recognizing its value with regard to enhancing infant growth and immunity and saving money.

I decided to breastfeed when I got pregnant... because breast milk has all the nutrients and immunity, so babies are healthy and strong. (P11)

I intended to breastfeed... breast milk is easy to digest... he will receive high immunity from breast milk...I got the information by asking nurses and doctors...They recommended that breast milk is good and should be provided exclusively from at least 6 months to 2 years. $(P 14)$

To prepare for breastfeeding while pregnant, they also managed themselves by seeking and receiving information and by consuming healthy foods.

\section{Seeking and receiving information}

The mothers sought to obtain as much information as they could about breastfeeding from various sources, including health care professionals, non-professionals, and the internet. Most indicated that they sought and received much information through a group learning session on breastfeeding, which was a part of their antenatal care. 
In antenatal care, the nurses advised watching the VDO with content on breast milk... It recommended that we should breastfeed for two years... breastfeeding for 6 months, then adding dietary supplements...The nurses gave information about how much nutrition breast milk contains. (P4)

Although the breastfeeding information provided by health care professionals was accurate, they still described a gap between knowledge and practice. I obtained very good knowledge...the nurses taught the group how to eat right and how to bring the baby to the breast, but it still didn't seem real...how to hold a baby by learning to hold a doll, but I felt different. (P13)

The mothers sought and received information from friends who breastfed their babies. This motivated them to breastfeed as well. One participant stated:

Friends who have children said breast milk is the best. Suckling milk from the breast promotes bonding. So, I wanted to give breast milk after giving birth. (P9)

In addition, they sought breastfeeding information from an application and the internet to obtain additional knowledge.

I downloaded an application... since I knew I was pregnant...This application is very good and has many details... It's like having a nurse there all the time. (P1)

\section{Consuming healthy foods}

The mothers consumed healthy foods that stimulate milk supply, such as dates and ginger, to prepare for lactation during pregnancy. They also drank ginger tea to boost breast milk based on information they received from relatives.

When I was 5 months pregnant, I ate dates sometimes. I ate dried dates or dates boiled with pandan leaves. It is so good for drinking. People in the maternity group on Facebook said that eating dates gave them a lot of milk. (P14) 

that it helps give a lot of milk. (P4)

\section{Phase 2: Overcoming the problem of insufficient milk supply}

Phase 2 started when the mothers gave birth and included the time when the babies were in the hospital. After the mothers gave birth to the preterm infants, they were confronted with separation from their infants and lack of skin-to-skin contact, causing lactation problems that led to insufficient milk supply.

After delivery, I saw my baby only for a second...the doctor told me my baby had to stay in the pediatric ward. He was preterm and his lungs weren't strong yet. I was afraid I wouldn't have any milk. Everything I'd read had said newborns have to be offered

breastfeeding within an hour or two after birth. (P4)

The mothers overcame the problem of insufficient milk supply by learning and practicing strategies to produce sufficient milk and exerting every effort toward achieving a sufficient milk supply.

\section{Learning and practicing strategies to produce sufficient milk}

The mothers received advice on how to produce sufficient milk by massaging from nurses and learned proper breastfeeding positioning and attachment. They learned to compress and massage the breasts and express breast milk for their infants every 2-4 hours. The nurses brought in towels with hot water and placed them on the breasts before the breast massage. The mothers tolerated learning to collect milk in a syringe for their babies:

The nurses taught me how to massage my breasts...they would bring me towels soaked in hot water... I tried to express milk. If I was able to get milk, I would collect it in a syringe. $(P 1)$ 
278

279

280

281

283

284

285

286

287

288

289

290

291

292

293

294

considerable amount of milk within a few days after massaging and expressing breast milk every 2-4 hours.

I expressed milk every 2-3 hours... the more frequently I expressed, the more I got... Once I was discharged, I was able to express about 1 ounce of milk. (P7)

In addition, the mothers learned to achieve proper breastfeeding positioning and attachment from pediatric nurses in the NS2 ward after their infant's condition had improved. The participants spent 30 minutes to one hour practicing every four hours or as the nurses were available:

I practiced positioning my baby at the breast in the NS2...Then they advised me about compressing my breasts and how to hold my baby at times when they could watch ...I practiced for an average of 30 minutes to 1 hour, depending on the nurses. (P15)

The mothers practiced the breastfeeding positions and attachment until they could execute them properly and the babies suckled well. Then, they could be discharged from the hospital:

When I knew how to do it, he suckled well. The baby was really good at it. When the baby suckled well, my milk kept flowing... and we were able to go home. (P13)

\section{Exerting every effort toward achieving a sufficient milk supply}

After learning and practicing techniques to produce sufficient milk, the mothers followed the nurses' advice. All of the mothers recognized that breastfeeding was beneficial for their babies. They wanted healthy babies, so they exerted every effort toward achieving a sufficient milk supply for them. The mothers mentioned that breast milk contains good nutrients, with more nutrition and immunity benefits than formula milk.

Breastfed babies are healthy, and breast milk has benefits. I'd like to breastfeed my baby for a year...but I'll be satisfied if we make it to six months. (P1) 
Mother's milk contains better immunity and more nutrients. If my baby were to drink formula milk, my baby would become bloated and so many other things...I want my baby to be healthy. (P2)

The mothers disciplined themselves to breastfeed, even though they had small amounts of milk at first. They woke up every night to pump milk to obtain a sufficient supply. Pumping milk was very painful, but the mothers were able to endure the pain because of their love for their child and desire to have a healthy infant:

When there wasn't milk and I was pumping, it hurt a lot until I thought about giving up.... If I was able to endure the pain when my baby was born, why wasn't I able to take just this? I endure this because I don't want my baby to be hurt or sick. (P8) It's in a mother's nature to produce milk. It's up to me to have the discipline to get it out... I have to pump, even at night...If I don't wake up to pump milk at night, there won't be enough milk...if I'm not strong... I won't be able to breastfeed successfully. (P4)

The mothers attempted to produce enough milk by breastfeeding frequently. They pumped milk so that they could give breastmilk at every feeding. After breastfeeding frequently, they had a greater milk supply. They felt glad to have sufficient milk for their babies: My baby has eight feedings a day...I try to pump for every feeding...I try to pump the amount my baby needs for each feeding... I try to pump as much milk as possible, and I don't limit the time...so, I have enough for my baby at each feeding. (P2)

When I pumped more frequently and on time, the milk increased... when I had enough milk, I cried. I was happy that I had enough milk and my baby was healthy. (P4)

While their babies were hospitalized, the mothers went to the hospital every day. They pumped milk and brought fresh breast milk to their babies, and they had appointments to practice breastfeeding. They also pumped breast milk at the lactation clinic at every hospital visit: 
I come to the hospital at times scheduled by the nurses to pump milk for my baby and practice breastfeeding... I have to come every day. When I come home, I have to pump milk for my baby. (P9)

I have to deliver milk to my baby every day. My baby needs fresh milk, as he is small and drinking fresh milk is best... I pump milk at night; when I get to the hospital, I pump again in the lactation clinic. (P5)

During breastfeeding, ten mothers had breast engorgement. This condition was relieved by massaging the breasts to express milk, with lactation nurses' assistance and independently: My breasts were engorged... I went to the lactation clinic. The nurses helped massage my breasts, so I felt better. (P4) My breasts were as hard as coconuts... I applied warm towels and brought a compress...I used the pump and hand compresses. It helped a lot. (P3)

Some mothers had babies with jaundice. Three babies had jaundice with glucose-6phosphate dehydrogenase deficiency (G6PDD). Most of the infants were treated by phototherapy and breastfeeding.

My baby was treated with phototherapy ...I brought him to breastfeed in the morning at 8 am and every 2-3 hours...then at night..., I pumped milk to bring to my baby...I wanted my baby to take milk and excrete a lot because the yellow substance comes out with urine and excrement. (P13)

My baby was jaundiced on the fifth day...she didn't get phototherapy. They told me to breastfeed her frequently. (P3)

In addition, the mothers resolved issues with ineffectively suckling babies by stimulating them to suckle by touching them, speaking with them, expressing milk into their mouth and stroking their cheeks. They tried to breastfeed until their babies suckled well: 
Once my baby had latched on, he would fall asleep...the nurse told me to stroke my baby's cheeks and talk to him...after stroking my baby's cheeks, I talked to him and he suckled some and slept some. (P9) Most of the time, my baby slept. He didn't suckle well. The nurse told me to try to wake my baby by speaking to or unwrapping my baby...my baby started to suckle well on the last day before coming home. (P15)

\section{Phase 3: Managing to continue breastfeeding}

Phase 3 started when the infants were discharged and lasted from when the babies went home to 6 weeks postpartum. The mothers recognized that breast milk is good for the health of the infant. However, at home, the mothers encountered lactation problems and could not consult with health care professionals; these problems included sleepiness, refusal to breastfeed and crying on the part of the infant.

Breast milk has so many benefits. It helps with bowel movements, but formula milk makes it difficult for babies to have bowel movements. My baby is healthy. (P11)

When we came back home, my baby was always asleep. I had to wake my baby up to breastfeed. (P4)

My baby wouldn't suckle, no matter what I did. ...I tried to get my baby to latch on, but my baby wouldn't. When I tried too hard, my baby struggled and cried. (P2)

Therefore, the mothers managed to continue breastfeeding by solving breastfeeding problems, boosting breast milk supply, adapting to daily activities and being committed to breastfeeding. Their strategies are discussed below:

\section{Solving breastfeeding problems}

The mothers had sore nipples because the babies mouthed at the nipples without deeply suckling over the areola. They solved this problem by stimulating their babies to open wide. 
375 They modified the breastfeeding position for attachment following the advice given to them by

376

377

378

379

380

381

382

383

384

385

386

387

388

389

390

391

392

nurses in the hospital:

I wasn't able to attach...My baby cracked my nipples...I tried to get my baby to suck deeply on the areola. (P3)

I think my nipples hurt...it's probably because my baby can't suckle up to the areola. I feel that... if I had my baby open wide and put it in his mouth, he would push it out after a while. He would take only the nipple...I took him off and gave it to him again. (P17)

\section{Boosting breast milk supply}

The mothers received advice from nurses, colleagues, and their family members to drink

herbal teas and avoid prohibited foods to boost breast milk supply for continued breastfeeding.

They drank ginger and banana blossom tea. They also drank a Northern Thai herb tea called Mai

Nomnang (xantolis) instead of water to increase milk production. After drinking, they felt their

breasts were full:

Most of the time, I have ginger tea...like drinking it in place of water... I drink when I feel thirsty and after pumping milk. I always drink it after waking up. I just drink it all the time. (P6)

Mai Nomnang is effective, because I had less milk and my breasts seemed empty while I was brewing it... When my aunt brewed some for me...I felt like I had milk flow. (P8)

The mothers avoided drinking cold water. They consumed only certain kinds of foods. If they did not follow a proper diet for an extended period, they might have insufficient milk for their babies:

I'd have no milk if I drank cold water because my body would be cold...So, I drank warm water before and after breastfeeding. I've mostly had rice porridge and boiled vegetables. I haven't had anything fried. I add pork without sauce... If I'd kept eating 
400 (P1)

\section{Adapting to daily activities}

The mothers adapted their activities in daily life to continue breastfeeding by managing their time to perform activities in a variety of situations. They made adjustments by sleeping in the daytime, doing activities while their babies were sleeping and asking family members to support them.

I didn't really get any sleep and my body was tired...I was tired in those early days.... I took some daytime naps, too. (P5)

If I'm alone, it's kind of hard...Things like showering, eating or doing laundry have to wait until the baby is asleep. Then I can do it. (P13) My mom manages all of the housework, diapers and other laundry. I feed the baby. Once my baby goes to sleep, I get to rest, too. (P15)

\section{Being committed to breastfeeding}

The mothers persevered with breastfeeding, despite their fatigue. They endured sleeping less because they were happy to breastfeed their babies. They felt rejuvenated when they saw their babies' faces and responses. Their babies were healthy while receiving breastfeeding. Hence, the mothers were committed to breastfeeding because of their love for their babies: Breastfeeding causes a child to have good health to avoid severe illnesses and being admitted to a hospital. (P13)

I feel good that I'm breastfeeding. I'm tired, but I have to endure this. I can't do anything about it. I have to endure for my baby because I love him... At night, my baby sleeps longer, for three hours. When he wakes up, he is hungry. He is very cute in my opinion (smiles and laughs). (P8) 

but I'm having fun. I feel that I'm doing all of this for something. (P3)

In summary, the mothers of late preterm infants adopted a maternal role of "striving for sufficient milk to have a healthy baby". A grounded theory approach was used to reach a preliminary stage of theory-construction. This enabled the researchers to define the phenomenon of producing sufficient milk as a process of great effort and exertion with respect to correct gestures, timing, facial expression, and offering the breast for infants to breastfeed. A theoretical diagram was developed regarding provision of adequate milk supply to breastfeed babies for improved health, composed of three categories: 1) preparing for breastfeeding, 2) overcoming the problem of insufficient milk supply, and 3) managing to continue breastfeeding. The process of producing sufficient milk helped participants achieve successful exclusive breastfeeding and improved health of their infants.

\section{Discussion}

Our findings highlight the core category of striving for sufficient milk to have a healthy baby. In a grounded theory study, according to the symbolic interactionist perspective, social interactions with others and the sociocultural environments encountered influence individuals' interpretations [2]. The theme of "Striving for sufficient milk to have a healthy baby" emerged from social interactions with others and the sociocultural environments of the first-time mothers of LPIs. During interactions, they expressed the thoughts, emotions, needs, and management processes leading to their actions or behaviors. This finding can be explained by the fact that the mothers of the LPIs entered the maternity world once they became aware of their pregnancies. They took on the maternal role. They demonstrated their behaviors for having healthy babies through gestures, timing, facing, and offering their breasts to breastfeed. 
At first, when the mothers learned that they were pregnant, they prepared for

449

450

451

452

453

454

455

456

457

458

459

460

461

462

463

464

465

466

467

468

469

470

471

472

breastfeeding by seeking and receiving information from nurses, friends, and the internet and by consuming healthy foods during pregnancy. This finding is consistent with that of Bryant et al., who found that women sought information during pregnancy from various sources, including friends, family, and the internet [3]. Interestingly, the mothers in the study consumed ginger tea and dates to promote their milk supply during pregnancy. This finding was in contrast with a literature review in which women consumed foods and herbs to increase their milk production during the postpartum period [4].

In the study, the mothers had preterm births, a situation they had not expected. At the time, they were separated from their infants by hospital protocol for caring for preterm infants. LPIs are typically healthier and suckle and swallow more effectively than early preterm infants [18], despite being separated from their mother. However, LPIs have more difficulty latching, suckling, and swallowing than full-term infants [1]. They do not have stimulation to initiate breastfeeding and skin-to-skin contact to promote breastfeeding. Hence, oxytocin, which is an essential hormone for triggering milk flow or the milk ejection reflex, is not released as it normally would be during breastfeeding [20]. Separation and a lack of skin-to-skin contact interrupt the breastfeeding process and lead to insufficient milk. Maastrup et al. (2014) found that early initiation of breast milk pumping before 12 hours postpartum may increase breastfeeding rates. The mothers in our study learned how to massage the breasts, express milk, and collect milk to produce a milk supply during separation from their babies until their babies were ready to breastfeed [21]. Jose et al. (2019) reported that breast massage is effective in increasing breast milk volume among mothers of premature neonates [16].

Furthermore, breastfeeding is a new experience, and first-time mothers experience difficulty maintaining milk supply. Our findings agree with those of Demirci et al. (2015) and Cescutti-Butler (2017), who reported that preterm mothers faced breastfeeding problems and 
473 difficulty maintaining their milk supply [9,8]. The mothers in this study breastfed frequently,

474 every 2-3 hours, to achieving a sufficient milk supply. This finding is similar to that of Sarapat

475 et al. (2017), who reported that mothers stimulated and expressed every 2-3 hours to maintain a

476 sufficient milk supply [25]. The mothers in this study relieved engorged breasts by massaging

477 their breasts, expressing milk, and pumping to remove milk. The management of breast

478 engorgement in this study is in accordance with a systematic review on treatment for breast

479 engorgement by Mangesi and Zakarija-Grkovic (2016) [22].

480 At home, the mothers encountered lactation problems but could not consult with health

481 care professionals. They dealt with these problems by themselves. They modified the baby's

482 position to allow good attachment to resolve cracked nipples. Yilak et al. (2020) reported that

483 poor positioning is a sign of ineffective breastfeeding techniques, potentially causing nipple pain

484 [32]. Incorrect suckling causes the mother's nipples to crack, leading to inadequate milk

485 production. In addition, the mothers in this study sought ways to boost their breast milk supply.

486 They drank herbal teas (ginger, banana blossom, and Mai Nomnang tea) to ensure sufficient milk

487 production, as advised by nurses, colleagues, and family members. Herbs (banana blossoms and 488 ginger) are used in Thailand to stimulate breast milk production and have long been widely 489 popular. Traditionally, herbs have been used in cooking or have been boiled to make teas [33]. 490 During breastfeeding, the mothers in this study consumed only certain kinds of foods and 491 avoided cold water to ensure that they had an adequate milk supply for their babies. This finding 492 is consistent with that of a study of traditional beliefs in China in which cold foods such as pork 493 liver soup, cock, and cuttlefish were found to be prohibited foods thought to decrease breast milk 494 production [28].

According to the findings, the mothers adapted their daily activities to continue 496 breastfeeding, such as by eating and bathing while their babies were sleeping, and asked their 497 family members to help them with housework while they were breastfeeding. The findings agree 
with the results of Flacking et al. (2007) that mothers struggle to balance life responsibilities while dealing with uncertain breastfeeding progress and muted feeding cues [10]. The mothers of the LPIs were committed to breastfeeding. They felt good about breastfeeding when they saw their babies' faces and responses, even though they felt fatigued. This finding is in accordance with that of Sarapat et al. (2017) [25], who showed that mothers were delighted when their babies fed at their breasts, and with the findings of Kair et al. (2015), who showed that breastfeeding is a beautiful bonding experience [17].

\section{Limitations}

The study was conducted at a university hospital in Chiang Mai, Thailand. The findings from this study are representative of a small group of first-time mothers of LPIs and cannot be generalized. Further research is needed using different types of triangulation methods to validate the findings in other groups across different cultures and societies.

\section{Conclusion}

This research has highlighted a specific aspect of mothers' experience of having an LPI, thereby providing new insights and contributing to a greater understanding of the breastfeeding experiences of such mothers. The aim of this study was to describe the experiences and management of exclusive breastfeeding among first-time mothers of LPIs. Using the grounded theory method [11], data analysis revealed that the experience and management of exclusive breastfeeding among first-time mothers of LPIs had three phases and a core category of striving for sufficient milk to have a healthy baby. This process describes the experience of first-time mothers with LPIs who adjusted to exclusive breastfeeding in order to have healthy babies.

The first of the three phases was preparing for breastfeeding, which began when the women became aware they were pregnant. The duration of Phase 1 extended from pregnancy to 
523 birth. Phase 2 was focused on overcoming the problem of insufficient milk supply, which began

524 when the mothers had preterm births and continued until their babies were discharged home.

525 Phase 3 was managing to continue breastfeeding, which began when the infants were discharged 526 home and lasted up to 6 weeks postpartum. From Phase 1 to Phase 3, the management process of 527 the first-time mothers of the LPIs was focused on striving for sufficient milk to have a healthy 528 baby. Thai first-time mothers of LPIs did everything they could to succeed in exclusively

529 breastfeeding their babies. They were resourceful and applied many strategies as they

530 endeavored to have an adequate milk supply to have a healthy infant.

532 that support first-time mothers of LPIs in successfully exclusively breastfeeding their babies.

533 Health care professionals play important roles in supporting these mothers by mobilizing social

534 support networks and encouraging breastfeeding. Moreover, nursing interventions can help 535 mothers achieve a sufficient milk supply. It is our hope that this study can be a useful 536 contribution to the existing literature on exclusive breastfeeding among first-time mothers of 537 LPIs.

Furthermore, the findings can be used as baseline data to encourage health care policy539 makers to enact policies that meet the needs of mothers, particularly those of LPIs who intend to 540 exclusively breastfeed. Specifically, the findings will help Thai policy-makers and health care 541 professionals understand the process of successfully striving to have a sufficient milk supply for 542 a healthy LPI, particularly when exclusive breastfeeding is involved. Group support, effective 543 mentorship programs, and national follow-up services for resource utilization targeting 544 breastfeeding mothers of LPIs could be offered as standard care in Thai hospitals. With this 545 study, health care professionals can reshape extant policies in ways that promote exclusive 546 breastfeeding for mothers of LPIs. By doing so, the health care community can provide support 547 for these mothers to exclusively breastfeed their babies to achieve good health. 
549 LPIs: Late preterm infants; WHO: World Health Organization; UNICEF: United Nations

550 Children's Fund; NICU: Neonatal Intensive Care Unit

551

552 Declarations

553 Ethics approval and consent to participate

554 Permission to conduct the study was obtained from the Institutional Ethics Review Board

555 Committee, Faculty of Nursing, and the Faculty of Medicine, Chiang Mai University, No. 2561-

556 EXP065 and No. 2561-05865. Potential participants were informed about the purpose and

557 process of the research and given oral and written guarantees of anonymity and confidentiality. It

558 was clarified that they could refuse to participate in or withdraw from the study at any time. All

559 participants signed informed consent forms, which included permission to record the interviews.

560 All interview transcripts were kept confidential and anonymous, and only the advisory

561 committee could check them for review purposes.

562

563 Consent for publication

564 Consent for the use of the qualitative data and for publication was obtained from each participant 565 before each interview.

566

567 Availability of data and materials

568 The datasets generated and/or analyzed during the current study are available from the 569 corresponding author upon reasonable request.

570

571 Competing interests

572 The authors declare that they have no competing interests. 
574 575 576 577 578

579 580

581

582

583

584

585

586 587 588 589 590 591 592

593

$594{ }^{1}$ Faculty of Nursing, Chiang Mai University, Chiang Mai, 50200, Thailand

$595 \quad{ }^{2}$ Boromarajonani College of Nursing, 10400, Bangkok, Thailand

$596{ }^{3}$ Center of Excellence in Nursing, Chiang Mai University, Chiang Mai, 50200, Thailand

\section{Author contributions}

Study Design: RC, KK, JC, NC

Data Collection: RC

Data Analysis: RC, KK

Manuscript Preparation: RC, KK, JC, NC

Study Supervision: KK, JC, NC

\section{Acknowledgments}

The authors would like to thank Boromarajonani College of Nursing, Bangkok, the Graduate School, and Office of Research Administration, Chiang Mai University, for funding and all the mothers of late preterm infants who took part in this study. This research work was partially supported by Chiang Mai University.

\section{Authors' information}

R. CHOIJORHOR ${ }^{1,2}$, RN, PhD, K. KANTARUKSA ${ }^{1}, \mathrm{RN}, \mathrm{PhD}, \mathrm{J}_{\text {. CHOTIBANG }}^{1,3}, \mathrm{RN}, \mathrm{PhD} \&$ N. CHALOUMSUK ${ }^{1}, \mathrm{RN}, \mathrm{PhD}$ 
598 Correspondence Address: Jutamas Chotibang, RN, PhD, Affiliation Center of Excellent in 599 Nursing, Faculty of Nursing, Chiang Mai University, 110/406 Intrawaroros Road, Suthep 600 District, Mueang, Chiang Mai, 50200, Thailand.

601 Tel: 66-53-93-5024, Fax: 66-53-21-7145, E-mail: jutamas.c@cmu.ac.th.

602

603

604

605

606

607

608

609

610

611

612

613

614

615

616

617

618

619

620

621

\section{References}

[1] Ahmed AH, Sands LP. Effect of pre-and postdischarge interventions on breastfeeding outcomes and weight gain among premature infants. Journal of Obstetric, Gynecologic \& Neonatal Nursing. 2010;39(1):53-63. https://doi.org/10.1111/j.15526909.2009.01088.x

[2] Aldiabat KM, Navenec L. Philosophical roots of classical grounded theory: Its foundations in symbolic interactionism. Qualitative Report. 2011;16(4):1063-80. https://doi.org/10.46743/2160-3715/2011.1121

[3] Bryant J, Waller AE, Cameron EC, Sanson-Fisher RW, Hure AJ. (2019). Receipt of information about diet by pregnant women: A cross-sectional study. Women and Birth. 2019;32(6):e501-e7.

[4] Budzynska K, Gardner ZE, Dugoua J-J, Low Dog T, Gardiner P. Systematic review of breastfeeding and herbs. Breastfeeding Medicine. 2012;7(6):489-503.

[5] Bureau of Health Promotion, Department of Health. Report A.D. 2014. 2015. Nonthaburi: Ministry of Public Health (in Thai). (Internet). 2017 (cited 23 January 2017). Available from: http://hp.anamai.moph.go.th

[6] Bureau of Reproductive Health, Department of Health. Mother's Guide to Postpartum and Baby Care: For Moms. 2019. Nonthaburi: Ministry of Public Health (in Thai). (Internet). 2019 (cited 10 January 2020). Available from https://pubhtml5.com/wrfu/kwrz/basic 
[7] Cartwright J, Atz T, Newman S, Mueller M, Demirci JR. Integrative Review of Interventions to Promote Breastfeeding in the Late Preterm Infant. Journal of obstetric, gynecologic, and neonatal nursing: JOGNN. 2017;46(3):347-56.

\section{https://doi.org/10.1016/j.jogn.2017.01.006}

[8] Cescutti-Butler L, Hewitt-Taylor J, Hemingway A. Powerless responsibility: A feminist study of women's experiences of caring for their late preterm babies. Women and Birth. 2020;33(4):e400-e8. https://doi.org/10.1016/j.wombi.2019.08.006

[9] Demirci JR, Happ MB, Bogen DL, Albrecht SA, Cohen SM. Weighing worth against uncertain work: the interplay of exhaustion, ambiguity, hope and disappointment in mothers breastfeeding late preterm infants. Maternal \& child nutrition. 2015;11(1):5972. https://doi.org/10.1111/j.1740-8709.2012.00463.x

[10] Flacking R, Ewald U, Starrin B. "I wanted to do a good job": experiences of 'becoming a mother' and breastfeeding in mothers of very preterm infants after discharge from a neonatal unit. Social Science \& Medicine. 2007;64(12):2405-16.

[11] Glaser BG, Strauss AL. The discovery of grounded theory: strategies for qualitative research. New York: Aldine Publishing Company; 1967.

[12] Glaser BG. Basic of grounded theory analysis. Mill Valley: Sociology Press.;1992.

[13] Glaser BG. Doing grounded theory: Issues and discussions. Mill Valley: The Sociology Press.;1998.

[14] Hackman NM, Alligood-Percoco N, Martin A, Zhu J, Kjerulff KH. Reduced breastfeeding rates in firstborn late preterm and early term infants. Breastfeeding Medicine. 2016;11(3):119-25.

[15] Holloway I, Galvin K. Qualitative research in nursing and health care (4th ed.). London: Blackwell; 2017. 
[16] Jose S, D'Souza SR, Sreedevi C. Effect of breast massage on breast milk volume and experience on the expression of breast milk among mothers of preterm neonates. Manipal Journal of Nursing and Health Sciences. 2019;5(2):6-11. Available from: https://impressions.manipal.edu/mjnhs/vol5/iss2/2

[17] Kair LR, Flaherman VJ, Newby KA, Colaizy TT. The experience of breastfeeding the late preterm infant: a qualitative study. Breastfeeding medicine. 2015;10(2):102-6. https://doi.org/10.1089/bfm.2014.0121

[18] Krol KM, Grossmann T. Psychological effects of breastfeeding on children and mothers. Bundesgesundheitsblatt-Gesundheitsforschung-Gesundheitsschutz. 2018;61(8):977-85.

[19] Lau C, Smith E. A novel approach to assess oral feeding skills of preterm infants. Neonatology. 2011;100(1):64-70. https://doi.org/10.1159/000321987

[20] Lawrence RA., Lawrence RM. Breastfeeding: A guide for the medical profession (8th ed.). Maryland Heights, MO: Mosby/Elsevier; 2016.

[21] Maastrup R, Hansen BM, Kronborg H, Bojesen SN, Hallum K, Frandsen A, et al. Factors associated with exclusive breastfeeding of preterm infants. Results from a prospective national cohort study. PloS one. 2014;9(2):e89077. https://doi.org/10.1371/journal.pone.0089077

[22] Mangesi L, Zakarija-Grkovic I. Treatments for breast engorgement during lactation. Cochrane Database of Systematic Reviews. 2016(6). https://doi.org/10.1002/14651858.CD006946.pub3

[23] Martin J, Osterman M. Describing the increase in preterm births in the United States, 20142016. NCHS Data Brief. 2018;(312):1-8. Available from: https://www.cdc.gov/nchs/products/databriefs/db312.htm

[24] National Statistical Office and United Nations Children's Fund. Thailand Multiple Indicator Cluster Survey 2015-2016, Final Report. NSO and UNICEF, Bangkok. (Internet). 2016 

(cited 30 August 2017). Available from: https://www.unicef.org/thailand/sites/unicef.org.thailand/files/201806/Thailand_MICS_Full_Report_EN_0.pdf

[25] Sarapat P, Fongkaew W, Jintrawet U, Mesukko J, Ray L. Perceptions and Practices of Parents in Caring for their Hospitalized Preterm Infants. PRIJNR [Internet]. 2017;21(3):220-33. Available from: https://he02.tcithaijo.org/index.php/PRIJNR/article/view/78177

[26] Semenic S, Loiselle C, Gottlieb L. Predictors of the duration of exclusive breastfeeding among first-time mothers. Research in nursing \& health. 2008 Oct;31(5):428-41. https://doi.org/10.1002/nur.20275

[27] Walker M. Breastfeeding Management for the Clinician: Using the Evidence. (4 ${ }^{\text {th }}$ ed). Sudbury, Massachusetts: Jones and Bartlett Learning; 2016.

[28] Wang Q, Fongkaew W, Petrini M, Kantaruksa K, Chaloumsuk N, Wang S. An Ethnographic Study of Traditional Postpartum Beliefs and Practices among Chinese Women. PRIJNR [Internet]. 2019;23(2):142-55. Available from: https://he02.tcithaijo.org/index.php/PRIJNR/article/view/118488

[29] World Health Organization. Breastfeeding. (Internet). 2020a (cited 30 August 2020). Available from: https://www.who.int/health-topics/breastfeeding\#tab=tab 1.

[30] World Health Organization, and The United Nations Children's Fund. Global nutrition target 2025: Breastfeeding Policy Brief. (Internet). 2014 (cited 30 August 2020). Available from: https://www.who.int/nutrition/publications/globaltargets2025_policybrief_breastfeeding len/

[31] World Health Organization. Infant and young child feeding. (Internet). 2020b (cited 30 August 2020). Available from: https://www.who.int/en/news-room/fact$\underline{\text { sheets/detail/infant-and-young-child-feeding }}$ 
697

698

699

700

701

702

703

704

705

706

707

708

709

710

711

712

713

714

715

716

717

718

719

720

721

722

723

724

725

726

727

728

729

730

731

732

733

734

735

736

[32] Yilak G, Gebretsadik W, Tadesse H, Debalkie M, Bante A. Prevalence of ineffective

breastfeeding technique and associated factors among lactating mothers attending public health facilities of South Ari district, Southern Ethiopia. PloS one. 2020;15(2):e0228863. https://doi.org/10.1371/journal.pone.0228863

[33] Yimyam S. Galactagogue herbs. Nursing Journal. 2018;45(1):133-45 (in Thai). Available from: https://he02.tci-thaijo.org/index.php/cmunursing/article/view/136183

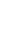

4

.

7


Fig.1 The process of "striving for sufficient milk to have a healthy baby".

\section{Table 1: Examples from the interview guideline}

What was your experience during pregnancy?

What were your postpartum experiences during your stay in the hospital?

How about your breastfeeding experience?

- How do you feel about breastfeeding?

- How does breastfeeding affect your life? How do you feel about this effect?

What are the needs (encouragement/support) during breastfeeding?

- From whom? How much support do you want? For what reasons?

How do you manage to exclusively breastfeed?

Table 2: Demographic Characteristics of the Participants $(\mathrm{N}=17)$

\begin{tabular}{lcc}
\hline \multicolumn{1}{c}{ Characteristics } & Number & Percentage \\
\hline Age (years) & 10 & 58.82 \\
$21-30$ & 7 & 41.18 \\
$31-37$ & 2 & 11.76 \\
Educational Level & 3 & 17.65 \\
Secondary School & 11 & 64.71 \\
Vocational College & 1 & 5.88 \\
Bachelor's Degree & & \\
Master's Degree & & 47.06 \\
Occupation & 7 &
\end{tabular}


Housewife

Merchant

Civil Servant

Government Employee

Type of Family

Extended

15

Nuclear

Number of Family Members (persons)

3-4

5-6

Family Monthly Income (baht)

$5001-10,000$

$10,001-15,000$

$15,001-20,000$

$20,001-25,000$

$25,001-30,000$
2

9

3

2

1

3

17.65

88.24

11.76

47.06

3

8

2
17.65

47.06

5.88

17.65

11.76 


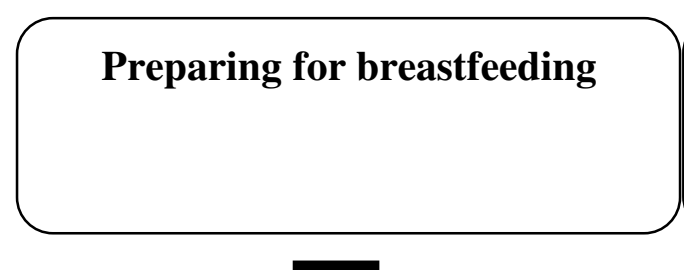

Overcoming the problem of
insufficient milk supply

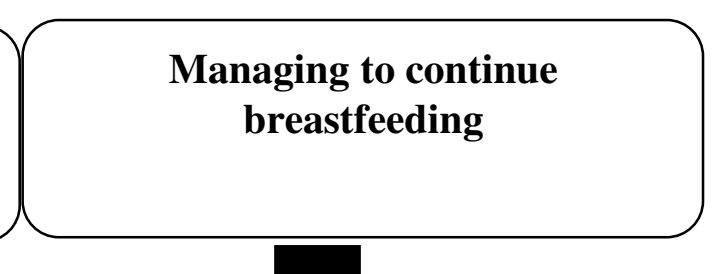

